\title{
Mechanics of Moisture for Polymers: Fundamental Concepts and Model Study
}

\author{
Xuejun Fan ${ }^{1,2}$ \\ ${ }^{1}$ Department of Mechanical Engineering \\ Lamar University, PO Box 10028 \\ Beaumont, Texas 77710, USA \\ ${ }^{2}$ Department of Engineering Mechanics \\ South China University of Technology \\ Guangzhou 510640, People's Republic of China \\ Tel: 409-880-7792; Fax: 409-880-8121 \\ e-mail: xuejun.fan@lamar.edu
}

\begin{abstract}
Reliability issues associated with moisture have become increasingly important as advanced electronic devices demand for new materials, more function integration and further miniaturization. However, the fundamentals of the mechanics of moisture transport and the subsequent failures in polymers have not been investigated extensively. This paper is devoted to some fundamental concepts of moisture absorption, diffusion, and swelling. The mathematical descriptions of moisture phase transition with temperature, and the governing equations for a deforming polymer with moisture effect are presented. The non-Fickian sorption behavior is discussed and a widely-used two-stage theory is introduced. It has been found that water sorption (immersion into liquid water bath) has very different transport mechanism at surface compared to the moisture sorption (moisture in air). The state of moisture within polymers and its interactions with polymer matrix, fillers and additives are discussed. It has been suggested that, in most of cases, the majority of absorbed water molecules neither show significant interaction with other water molecules nor with their environment. Those 'unbound' moisture stays in free-volumes and does not contribute to hygroscopic swelling. Swelling is caused by water molecules bound to the polymer matrix. Although the free volume fraction is usually in the range of $1 \%$ to $5 \%$, the pore size is always below nanometers and not detectable by the conventional measurement techniques such as SEM and mercury intrusion. An approximate estimate of free volume fraction using weight gain data is introduced. A vapor pressure model, which allows the whole-field vapor pressure calculations, is presented. Finally the governing equations for a deforming polymer with the consideration of moisture are developed.
\end{abstract}

\section{Introduction}

In microelectronics encapsulation and packaging, polymers are extensively used as adhesives, encapsulants, passivation and printed circuit boards. It has been long recognized that moisture plays a key role in the reliability of microelectronic packaging [1-4]. Moisture absorbed by polymer-based packaging materials can cause substantial changes in material properties, such as coefficient of thermal expansion (CTE), modulus, glass transition temperature ( $\mathrm{Tg})$, and viscoelastic behavior. In cured thermosets, moisture acts as a plasticizer, reducing the modulus and $\mathrm{Tg}$, and changing the thermal expansion characteristics of the material. For example, upon moisture saturation at $85^{\circ} \mathrm{C} / 85 \%$ relative humidity $(\mathrm{RH})$, the $\mathrm{Tg}$ of a cured no-flow underfill decreased by as much as $25^{\circ} \mathrm{C}$, whereas its room temperature modulus decreased by approximately $8 \%$ [5]. At elevated temperatures, moisture-induced hygroscopic swelling in packaging materials can cause a large increase in package stresses. For example, for some underfill materials, the hygroscopic swelling induced strain is comparable to the thermal strain caused by thermal expansion over a temperature range of $100^{\circ} \mathrm{C}$ [5-7]. At solder reflow temperatures, which are typically $230-260^{\circ} \mathrm{C}$, vaporization of residual moisture leads to a sharp buildup in vapor pressure, causing voiding, cracking, interfacial delamination, or "popcorn" failures in packages [8-10]. In addition, moisture can cause degradation of adhesion strength, which leads to a reduction of interfacial strength [9, 11-13]. Many failures in microelectronic packages can be traced back to moisture [1]. Reliability issues associated with moisture have recently become increasingly important as new materials such as waferlevel films are introduced and new failure mode after preconditioning test has been detected, such as cohesive failure within the film material $[8,10]$.

Unlike the mechanics of heat transfer and thermal stresses in polymer materials, the fundamentals of mechanics of moisture in polymers have not been investigated extensively. There exist published articles in the open literature but the terminologies and approaches presented in the literature are sometimes confusing. This paper is devoted to some fundamental concepts of moisture absorption, desorption, diffusion, swelling and moisture phase transition with temperature change. The non-Fickian diffusion behaviors and the treatment in engineering practices are presented. The difference in diffusion mechanisms between moisture sorption and water sorption is discussed. Porosity measurement and the estimate of free volume fraction are introduced. The moisture state within polymers and the linkage to the solubility and hygroscopic swelling are studied. A vapor pressure model considering phase transition with respect to temperature rise is introduced. Finally, a general 
framework of the governing equations for a deforming polymer with consideration of moisture is presented.

\section{Fickian and Non-Fickian Kinetics}

Fickian transport in polymers is described by

$$
\frac{\partial C}{\partial t}=D\left(\nabla^{2} C\right)
$$

This equation is known as the general diffusion law or Fick's second law of diffusion (Fick's law, for short). $C$ $\left(\mathrm{kg} / \mathrm{m}^{3}\right)$ is the penetrant concentration, $t(\mathrm{~s})$ the time, and $D\left(\mathrm{~m}^{2} / \mathrm{s}\right)$ is the diffusion coefficient or diffusivity of the moisture in the polymer. $D$ is the pivotal parameter determining the rate of transport. It may depend strongly on concentration $[14,15]$.

The temperature dependence of the diffusion constant can be described by the Arrhenius equation as follows

$$
D=D_{o} \exp \left(-\frac{E_{d}}{k T}\right)
$$

where $D_{o}$ is a pre-factor, $E_{d}$ is the activation energy, $k=1.38 \times 10^{-23} \mathrm{~J} / \mathrm{K}$ is the Boltzmann's constant, $T$ the absolute temperature. In some published papers, the minus sign inside the exponential function was omitted and a negative activation energy was used. It should be noted that the pre-factor and the activation energy constants are different across the glass transition temperature.

Fig.1 is a typical plot for the so-called Fickian absorption kinetics. Characteristic features of such curve are: 1. an initially linear plot of $M$ (weight gain) as a function of $\mathrm{t}^{1 / 2}$ (in one-dimensional diffusion), where $M$ is the mass of moisture; 2). With increasing $t$, the absorption curve smoothly levels off to a saturation level $M_{\infty}$. The saturated moisture concentration $C_{\text {sat }}$ is often used to describe the capacity of moisture absorption at given humidity and temperature conditions as follows

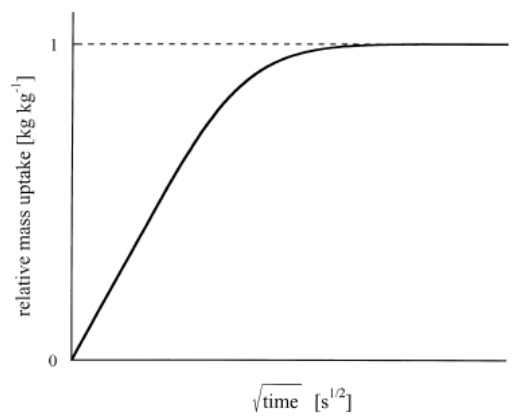

Fig.1. Schematic of a typical Fickian absorption curve. The relative weight gain $M(t) / M_{\infty}$ is plotted as a function of the square root of time $t$.

$C_{s a t}=M_{\infty} / V$

where $V$ is the volume of the sample, over which moisture mass is $M_{\infty}$. Fickian diffusion in polymers is an ideal case of moisture transport, corresponding to free diffusion of moisture without interference of polymer chain rearrangement, i.e., structural relaxation. Whether deviations from ideal Fickian behavior occur depends on the rate of relaxation compared to that of diffusion. If polymer relaxation is much faster than moisture diffusion, diffusion is followed by instantaneous response of the system, resulting in Fickian behavior.

Vrentas et al. [16] introduced a diffusion Deborah Number $(\mathrm{DEB})_{\mathrm{D}}$ to indicate the presence of non-Fickian effects during absorption experiments. This number is defined as

$$
(D E B)_{D}=\frac{\lambda_{m}}{\theta_{D}}
$$

where $\theta_{\mathrm{D}}$ (s) is the characteristic time of penetrant diffusion and $\lambda_{\mathrm{m}}$ (s) can be considered as the characteristic time of polymer relaxation processes. $\theta_{\mathrm{D}}$ is proportional to the square of polymer thickness. Calculation of the diffusion Deborah number is described in [16]. Vrentas et al. suggested that three different zones in a C-T diagram can be distinguished, separated by lines of constant Deborah number, as shown in Fig. 2.

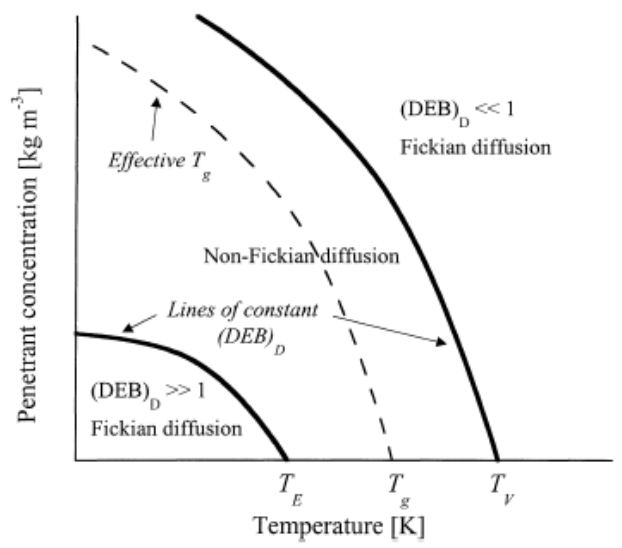

Fig.2. Schematic picture of the different zones of diffusion, separated by lines of constant diffusion Deborah number $(\mathrm{DEB})_{\mathrm{D}}$, as related to moisture concentration and temperature. $\mathrm{T}_{\mathrm{E}}$ is the temperature below which pure polymer acts like an elastic solid, $\mathrm{Tg}$ the glass transition temperature, and $\mathrm{T}_{\mathrm{V}}$ is the temperature above which pure polymer acts like a viscous fluid.

There are a number of non-Fickan diffusion kinetics [14], of which, the "two-stage" sorption is a frequently encountered non-Fickian type of absorption. The general appearance of the two-stage sorption is shown in Fig. 3, which is characterized by a rapid initial uptake to a quasiequilibrium, followed by a slower approach to a final, true equilibrium. In Fig. 3, the curve is Fickian from the start until the moment it starts to level off. However, instead of reaching the saturation level typical for Fickian absorption, the curve is extended through a non-Fickian part. Ultimately, saturation will be reached for all instances. A theory, satisfactorily describing the features of 'two-stage' sorption, has been proposed by Berens and Hopfenberg [17]. In their diffusion relaxation model they considered the absorption process to be composed of two 
phenomenologically independent contributions: a diffusion part $\mathrm{M}_{\mathrm{F}}(\mathrm{t})$ that is governed by Fick's law and a structural part $\mathrm{M}_{\mathrm{R}}(\mathrm{t})$, resulting from polymer relaxations. The total weight gain at time $t$ may be expressed as the linear superposition of these contributions, as follows

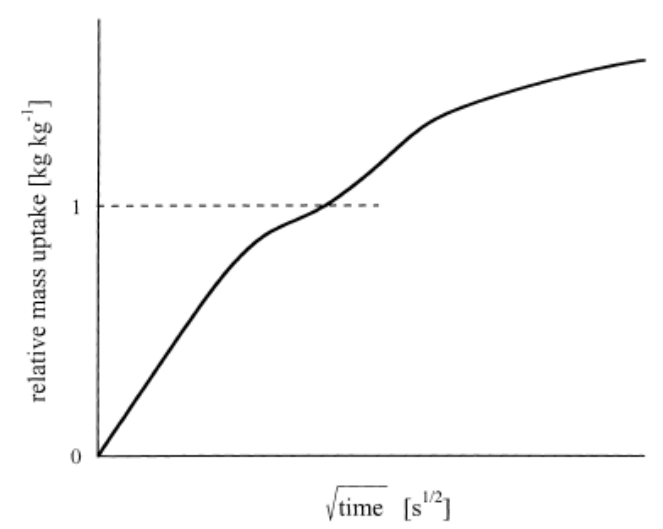

Fig.3. Schematic picture of a typical two-stage absorption curve. The relative weight gain $M(t) / M_{\infty}$ is plotted as a function of the square root of time. $M(t) / M_{\infty}=1$ for the (fictitious) Fickian saturation level.

$M(t)=M_{F}(t)+M_{R}(t)$

$M_{\mathrm{F}}(t)$ is given by solutions of the diffusion Eq. (1). It is assumed that more than one independent relaxation process is possible, so $M_{\mathrm{R}}(t)$ is given by

$$
M_{\infty}(t)=\sum_{i} M_{\infty, i}\left(1-e^{-k_{i} t}\right)
$$

where $\mathbf{M}_{\infty, \mathrm{i}}$ represents the equilibrium absorption due to the $i$ th relaxation process, and $k_{i}$ is the first-order relaxation constant of the $i$ th relaxation process. Originally, the model has been developed for diffusion in spherical particles, but it has also been applied to diffusion in polymer films, e.g. [18].

Bismaleimide-triazine (BT) resin/glass fiber laminates are commonly used as a substrate core material in microelectronic packaging. Their moisture absorption and diffusion behavior have a significant impact on package reliability. Fig. 4 plots the moisture weight gain for a thickness of $0.6 \mathrm{~mm}$ at $30^{\circ} \mathrm{C} / 60 \% \mathrm{RH}$. It clearly shows the two-stage moisture absorption. The material behaves initially as Fickian's behavior. However, the non-Fickian effect starts after the certain time.

Whether the non-Fickian effect should be considered depends on the duration of exposure time to moisture in field conditions or testing conditions. If the exposure time is within the period before polymer relaxation has taken place, Fick's law will describe the moisture diffusion very well. Otherwise non-Fickian's behavior must be accounted for. In semiconductor industry, the duration of moisture exposure at different humidity levels is usually 7-9 days. The non-Fickian effect depends not only the exposure time, but also on material's chemistry, geometry and humidity level, since the characteristic time of penetrant diffusion is function of the thickness of the sample.

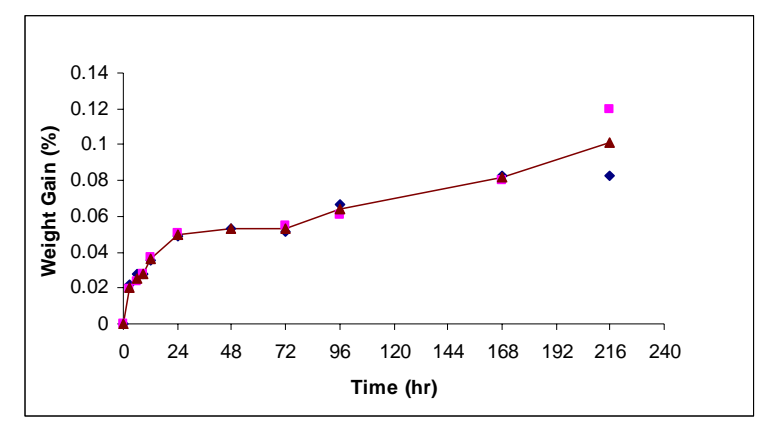

Fig.4. Moisture weight gain curve for a BT sample with the thickness of $0.6 \mathrm{~mm}$ subjected to $30^{\circ} \mathrm{C} / 60 \% \mathrm{RH}$.

\section{Water Sorption and Moisture Sorption}

When a polymer material is immersed into liquid water, the sorption process is referred to water sorption. For a material that is subjected to a humid air condition with a relative humidity less than (or equal to) $100 \%$, it is referred to moisture sorption process. The ambient moisture for water sorption is of course in water liquid form, but the moisture in air stays as vapor form. There are two distinct diffusion mechanisms involved in the transport of moisture: the transfer across the surface and the transfer through bulk. Once moisture is penetrated through the surface, a similar diffusion mechanism such as Fickian kinetics will follow in the bulk of materials. However, the diffusion mechanism across the surface may be very different and depends strongly on the state of ambient moisture and the chemistry of materials. For example, some coating material is water-resistant or water-proof, which can prevent water molecular from penetrating into the material. However, such a material may not be immune from the penetration of water vapor (see Fig.5). We performed an experiment on moisture sensitivity test for two groups of QFP packages [19]. One group of QFP packages was coated with a water-proof film to prevent water uptake, while the other group is as received. Two types of moisture preconditioning were applied: immersion into water with a constant temperature of $60^{\circ} \mathrm{C}$, and the placement in a humidity chamber at $60^{\circ} \mathrm{C} / 85 \%$ RH. Both sorption durations were 192 hours. The moisture weight gain data showed that the coated package did not have any weight change when subjected to the immersed water. However, the coated package gained almost the same amount of moisture compared to the uncoated packages when both were subjected to $60^{\circ} \mathrm{C} / 85 \% \mathrm{RH}$ condition. Further, the moisture sensitivity test indicated that the coated package at $60^{\circ} \mathrm{C} / 85 \% \mathrm{RH}$ preconditioning had the same failure rate with the uncoated in the same preconditioning conditions. The coated package immersed in the water did not show any failures. These results imply that some coating polymer films are very effective in isolating the water liquid from penetrating through the surface, but cannot stop water 
vapor uptake. More studies are needed to understand the surface diffusion mechanism. Throughout this paper, unless it is stated, the moisture absorption process is generally assumed.

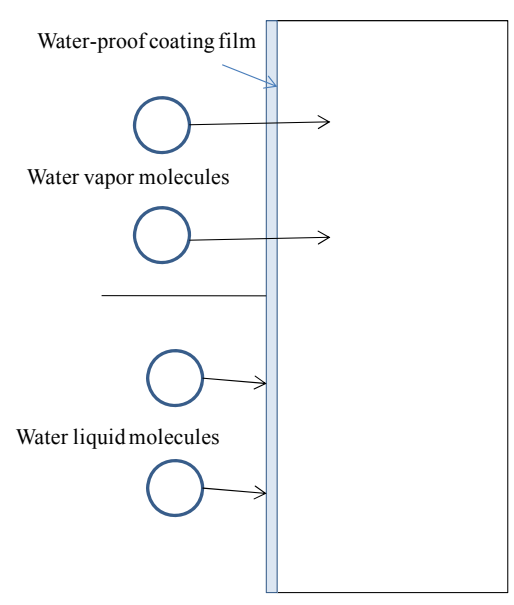

Fig.5. Schematic picture for a water-proof coating film, which cannot prevent water vapor from penetrating into the film.

\section{State of Moisture within the Bulk Polymers and Interactions with Polymers}

Once the moisture enters the polymer matrix, the moisture will be condensed into liquid water phase. For example, let us consider the moisture absorption for a typical mold compound in $85^{\circ} \mathrm{C} / 85 \% \mathrm{RH}$ condition. Assume that moisture diffusion follows Fickian kinetics with $M_{\infty}$ being the ultimate moisture weight gain for a sample volume of $V$. The saturated moisture concentration, $\mathrm{C}_{\mathrm{sat}}$, as defined in equation (3), can be calculated from a weigth gain test. A typical value of the $\mathrm{C}_{\mathrm{sat}}=12.5 \mathrm{mg} / \mathrm{cm}^{3}[20]$. The moisture vapor density at $85^{\circ} \mathrm{C} / 85 \% \mathrm{RH}$, i.e., $\rho_{\text {ext }}$, can be obtained from the saturated water vapor density at $85^{\circ} \mathrm{C} / 85 \% \mathrm{RH}$

$\rho_{\text {ext }}=0.85 \rho_{\mathrm{g}}=3.04 \mathrm{e}^{-4} \mathrm{~g} / \mathrm{cm}^{3}$

where $\rho_{g}$ is the saturated water vapor density at $85^{\circ} \mathrm{C}$ (obtained from the steam table). A simple comparison between the $\mathrm{C}_{\mathrm{sat}}$ and $\rho_{\mathrm{ext}}$ reveal that $\mathrm{C}_{\mathrm{sat}}=41 \rho_{\mathrm{ext}}$. If the free volume fraction is $5 \%$ of the total sample volume (to be discussed in next section), the actual moisture density in the free volume is then

$$
\rho=\text { free volume fraction } \times C_{\text {sat }}=820 \rho_{\text {ext }}=0.25 \mathrm{~g} / \mathrm{cm}^{3}
$$

This implies that the moisture inside the mold compound must be in the mixed water liquid/vapor phase. The diffusion process of moisture is the transport process of the moisture from ambient vapor phase to the condensed mixed liquid/vapor phase.

According to Duda and Zielinski's model [21], polymer volume is divided into three elements (see Fig. 6): 1. Occupied volume. This is basically the "van der Waals" volume and is the volume of the material without any free volume. This volume can be calculated from bond distances and non-bonded contact radii - the socalled "van der Waals" radii - and is a constant for all temperatures; 2. Interstitial free volume. This inaccessible free volume stems from the vibration energy of polymer bonds, and increases slightly with temperature; 3 . Hole free volume. This is the volume stemming from volume relaxation and plasticization upon heating and cooling of the polymer. This free volume is accessible for penetrant transport, and may be altered by absorption and desorption of penetrants. Changes in the total polymer volume are largely governed by changes in the hole free volume. Rubbery polymers attain equilibrium volume, since polymer relaxations are fast. Glassy polymers, however, are non-equilibrium liquids in which relaxations are slow. Consequently, glassy polymers may have larger volume than would be the case at equilibrium, since extra hole free volume is trapped in the polymer. Although polymers seem to be porous viewed in this way, Duda and Zielinski emphasize that volume trapped in a glassy polymer is continuously redistributed through the volume.

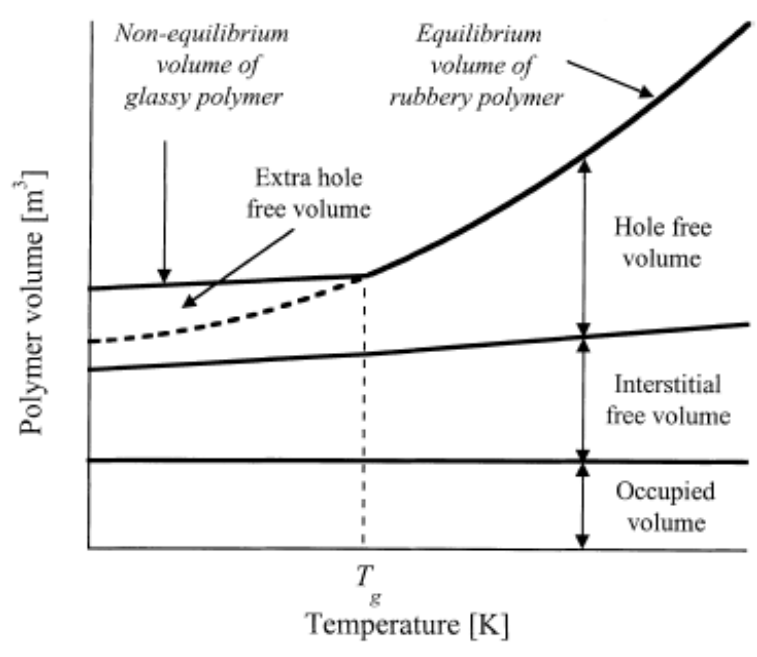

Fig. 6. Schematic picture of polymer volume as a function of temperature [21].

From Fig. 6, it can be seen that the free volume fraction is not sensitive to temperature when the temperature is below the $\mathrm{Tg}$. However, in the rubbery state, the free volume increases signficantly with temperature. This increase will be related to the saturated moisture content to be discussed in next section.

It has been suggested that the majority of absorbed moisture stays in free volume and free to move. This is referred as 'unbound' water liquid or vapor, which collects at micro- or nano- pores, free volumes, interfaces, and micro- or macro voids. More than $90 \%$ of the absorbed moisture stays in unbound water state [22]. Additionally, water can be immobilized within the material. This can happen in a varitey of ways. Because of strong tendancy for water to form hydrogen bonds (often called bound water), water molecules may congregate amongst themselves, forming a separate 
phase. Water may also be trapped at sites along the polymer chain due to physical and chemical interactions.

Many polymer materials used in electronic packaging consist of fillers. While most fillers are not used with the intent of altering the sorption and transport of water within polymer, this concomitant effect is almost always unavoidable. Much of the effect of the fillers on the transport properties within the matrix is governed by the degree of interaction between the polymer matrix and the filler at the interphase. In most composites, it is desirbale to have a strong adhesive bond between the two materials. The adhesive bonds can be of either a physical or physicochemical nature. Variations in the strength and nature of these bonds lead to three typical situations at the polymer/filler interface [23].

If the bonds are physcial, water can be prevented from reaching the interfacial regoin and the amount of water absorbed depends only on the epoxy fraction of the composites. If physicochemical bonds, such as hydrogen bonds, are involved, the polymer may find its movement restricted in the vicinnity of the filler. If this attraction is strong enough, a porton of the polymer matrix as well as the filler volume may be impermeable to the penetrating moistue.

If poor bonding exists at the interface, water transport may be enhanced by pathways open along the interface. In this case, the water is transported in the liquid state by capillary forces. Similar capillary transpoort may occur if microcracks and/or defects are present in the bulk of expoxy. Voids at the interfcae may increase the Langmuir sites of the composites, allowing higher equilibirum sorption. Water clustering in these voids can cause positive deviations from Henry's law [24].

The effect of interfacial bonding can be taken into account by comparing diffusion and sorption in neat polymer resins and composites. If, after correcting for the fiber content of the composite, there is no difference between the two, interphase effects may be negligible. This in general is found for glass/epoxy systems [25].

Fig.7 shows an example of moisture absorptiondesorption experiment for BT of $70 \mu \mathrm{m}$ thickness [5], where the temperature was kept at $60^{\circ} \mathrm{C}$ and the $\mathrm{RH}$ level was cycled between 0 and $60 \%$. During the first 60 minutes, a $0 \% \mathrm{RH}$ condition was imposed to drive any residual moisture out of the sample. This was followed by a $200 \mathrm{~min}$ of moisture absorption at $60 \% \mathrm{RH}$, then a 200 min desorption at $0 \% \mathrm{RH}$. This sorption-desorption cycle was repeated one more time, as shown in Fig.7. The subsequent absorption-desorption cycles were repeatable, i.e. the sample reached approximately the same saturated moisture level during sorption and it lost the same weight upon drying. This indicates that there is no chemical reaction between the water molecules and the material. The moisture absorption for most of polymer materials used in electronic packaging is a reversible process.

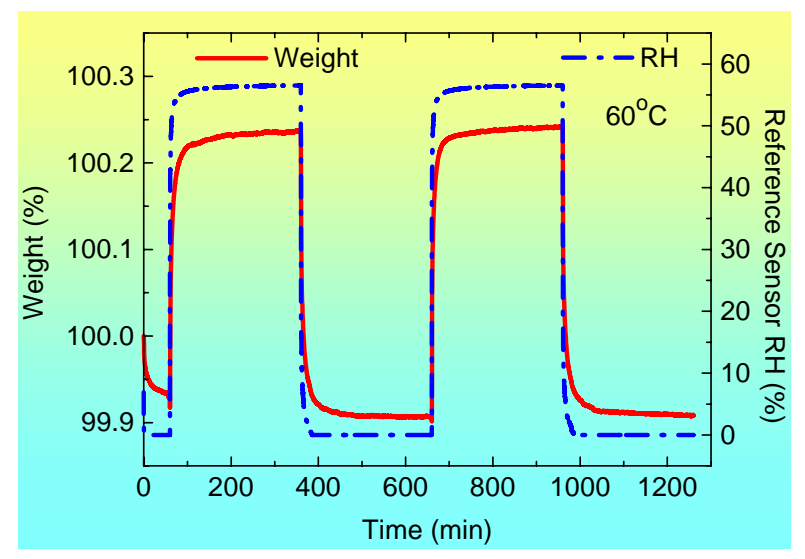

Fig.7. Moisture absorption-desorption experiment conducted using a sorption TGA at $60^{\circ} \mathrm{C}$ with the $\mathrm{RH}$ level cycled between 0 and $60 \%[5]$.

\section{Characterization of Porosity and Free Volume}

Porosity or free volume fraction of polymer is one of critical material properties related to the moisture absorption. Mercury intrusion porosimetry characterizes a material's porosity by applying various levels of pressure to a sample immersed in mercury [26]. The pressure required to intrude the mercury into sample's pores is inversely proportional to the size of pores. Mercury porosimetry is based on the capillary law governing liquid penetration into small pores. This law, in the case of a non-wetting liquid like mercury, is expressed by the Washburn's equation

$$
D=\frac{-4 \gamma \cos \theta}{P}
$$

where $\gamma$ is surface tension of mercury, $\theta$ is the contact angle between the mercury and the sample, $\mathrm{P}$ is the applied pressure, and $\mathrm{D}$ is the pore diameter, all in consistent units. The volume of mercury $\mathrm{V}$ penetrating the pores is measured directly as a function of applied pressure. This $\mathrm{P}-\mathrm{V}$ information serves as a unique characterization of pore structure. The Washburn equation assumes that all pores are cylindrical. Although pores or free volumes are rarely cylindrical in reality, this equation provides a practical representation of pore distributions, yielding very useful results for most applications. As pressure increases during an analysis, pore size is calculated for each pressure point, and the corresponding volume of mercury required to fill these pores is measured. These measurements taken over a range of pressures give the pore volume versus pore size distribution for the sample material. Mercury porosimetry can determine pore size distribution accurately. Comprehensive data provide extensive characterization of sample porosity and density. Available results include total pore volume, pore size distribution and pore diameter. In general, mercury porosimetry is applied over a capillary diameter range from $50 \mathrm{~nm}$ to $360 \mu \mathrm{m}$.

Several types of polymer materials in packaging applications, such as die-attach films, solder mask, and 
underfills, were tested using Mercury intrusion [27]. Those materials were also applied for moisture weight gain test at $85^{\circ} \mathrm{C} / 85 \% \mathrm{RH}$. The results showed that the relative weight gain ranged from $0.48 \%$ to $1.27 \%$ for those tested materials. However, as expected, no significant porosity down to $\sim 50 \mathrm{~nm}$ was observed for all the tested materials. This implies that the free-volumes, which are occupied by the absorbed moisture, is very small is size and the typical dimension is in nanometer range. The SEM analysis was also performed, and no appreciable pores were observed.

Further, the die-attach films were applied for a stackdie chip scale package. One of the die-attach films was very soft with a tensile modulus less than 10 or even 1 $\mathrm{MPa}$ at solder reflow temperature $[8,10,28]$. The moisture sensitivity test at level 3 [29] was performed. Cohesive delamination and cracking of the first layer die-attach film was observed using TSAM technique (see Fig. 8). Further by applying top-down lapping, the microstructure change of the film after reflow with moisture was captured, as shown in Fig. 9. Significant voiding of the film due to vapor pressure expansion was developed, even though the pore-size and free-volume size was not detectable before the reflow test.

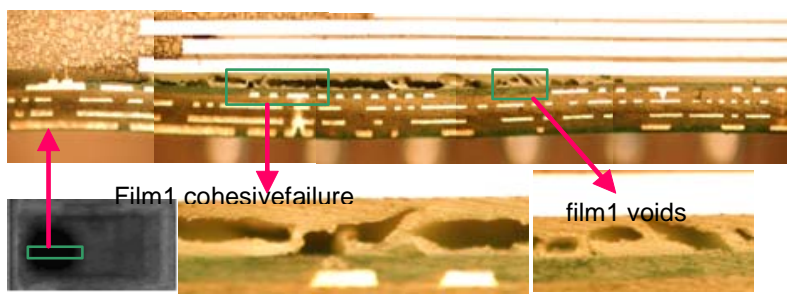

Fig.8. After moisture sensitivity test at level 3, massive cohesive delamination was seen in the first layer die attach film (film 1). The cohesive delamination is a consequence of void growth and coalescence induced by vapor pressure during the reflow process.

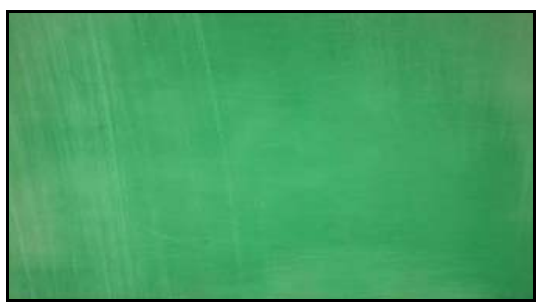

a)

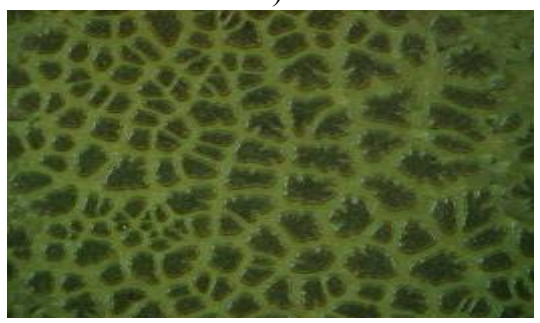

b)

Fig.9. A soft die-attach film in a stack-die chip scale package before and after reflow with moisture absorption.
An approximate estimate method to obtain the free volume fraction of polymers was proposed using moisture weight gain test [30]. Consider a material with a saturated moisture concentration $C_{\text {sat }}$ at the given temperature and humidity. If the moisture density in those pores or freevolumes is denoted as $\rho$ within a representative volume element (to be discussed later), the following relationship exists

$f_{0}=\frac{C_{\text {sat }}}{\rho}$

where $f_{0}$ is the initial free volume fraction.

Since the density of the liquid water is $1.0 \mathrm{~g} / \mathrm{cm}^{3}$, the moisture density in free volume, i.e., $\rho$ in equation (10), must be less than or equal to $1.0 \mathrm{~g} / \mathrm{cm}^{3}$,

$f_{0} \geq C_{\text {sat }}$

when the Csat uses the unit of $\mathrm{g} / \mathrm{cm}^{3}$. In general, the $C_{\text {sat }}$ depends on the relative humidity and temperature. It has been suggested that the water liquid will fill in the free volume completely at $100 \% \mathrm{RH}$. Therefore the initial free volume fraction can be estimated from a moisture weight gain test using $C_{\text {sat }}$ measurement data and extrapolated to the $100 \% \mathrm{RH}$. This estimate is a low-bound estimation since the moisture will be usually in the mixture of water and vapor in free-volumes. Table 1 lists the results of the free volume fraction for some commonly used plastic materials in IC packages. The results were based on equation (11) using the material property data measured by Galloway et al [31]. It showed that the free volume fraction is usually between $1 \%$ and $5 \%$.

As seen from Fig.6, the free-volume below $\mathrm{Tg}$ is usually insensitive to temperature. However, above the glass transition temperature, the free volume will increase with temperature significantly. If the glass transition temperature is greater than $100^{\circ} \mathrm{C}$, the $C_{\text {sat }}$ data are not available since a pressurized chamber with an in-situ measurement is required.

Table 1 Initial void volume fraction for some materials in IC packages

\begin{tabular}{lrrcc}
\hline Material & BT & $\begin{array}{c}\text { Die- } \\
\text { attach }\end{array}$ & $\begin{array}{c}\text { Mold } \\
\text { compound }\end{array}$ & $\begin{array}{c}\text { Solder } \\
\text { mask }\end{array}$ \\
\hline$f_{0}(\%)$ & 3.46 & 3.29 & 1.46 & 5.05 \\
\hline
\end{tabular}

\section{Hygroscopic Swelling}

Upon moisture absorption, polymer material will expand. For example, in some microelectronic packages encapsulated using commercially available molding compounds, the strain induced by the mismatch of the coefficients of hygroscopic swelling is nearly twice as much as the strain induced by the CTE mismatch over a temperature span of $\Delta \mathrm{T}=60{ }^{\circ} \mathrm{C}$ [5]. For other molding compounds, the hygroscopic mismatch induced strain 
ranges from one to nearly four times of the strain induced by the CTE mismatch over a $\Delta \mathrm{T}$ of $45^{\circ} \mathrm{C}$ for $\mathrm{T}>\mathrm{Tg}$ or over a $\Delta \mathrm{T}=100^{\circ} \mathrm{C}$ for $\mathrm{T}<\mathrm{Tg}$. For some underfill materials, the hygroscopic swelling induced strain is comparable to the thermal strain caused by thermal expansion over a temperature range of $100^{\circ} \mathrm{C}$ orders of magnitude! The typical values of the hygroscopic swelling range from $0.1 \%$ to $0.5 \%$. The volume change of the hygroscopic swelling corresponding to $0.3 \%$ can be expressed as

$$
\frac{\Delta V}{V}=0.3 \%
$$

where $\mathrm{V}$ is the total volume of the sample, and $\Delta \mathrm{V}$ is the volume change due to moisture absorption at a constant temperature.

On the other hand, if the material's free volume fraction is estimated as $3 \%$ as a representative value as follows

$$
f_{0}=\frac{V_{\text {free volume }}}{V}=3 \%
$$

It reveals that the volume change by hygroscopic swelling is only a small fraction of the total free volume (in this example it is $10 \%$ ). In the previous section, the estimate of the free volume fraction does not consider the effect of hygroscopic swelling. This example inferred that the estimate of free volume fraction using weight gain data without considering the material swelling give a good approximation if the material does not swell excessively. It also means that the majority of moisture absorption does not contribute to the material's swelling.

It has been suggested that swelling is caused by water molecules bound to the polymer matrix and not by the free water molecules [32]. Because the water molecule is polar, it is capable of forming hydrogen bonds with hydroxyl groups, thereby disrupting inter-chain hydrogen bonding with the net effect of increasing the intersegmental hydrogen bond length. This concept has been observed through spectroscopic methods. Specifically, in the study done by Vanderhart et al. [33] two distinct states of water were observed through nuclear magnetic resonance (NMR) spectroscopy of an epoxy molding compound used in electronic packaging. In another study, two distinct states of water were also observed in cellulose acetate polymers using infrared (IR) spectroscopy [34].

In order to characterize the swelling behavior, the coefficients of hygroscopic swelling (CHS) is often introduced as following

$$
\varepsilon_{\text {swelling }}=\beta C
$$

where $\beta$ is the coefficient of hygroscopic swelling and $\mathrm{C}$ is the moisture concentration. According to the above analysis, the formation of hydorgen bond with polymer materials causes the hygroscopic swelling of material, while the unbound water liquid/vapor fills in free volumes, which does not cause swelling if the vapor pressure is low at lower temperatures. Therefore, the above equation might needs to be modified if only a small fraction of moisture is responsible for the swelling of material. Instead of using the total moisture concentration in equation (12), the moisture concentration fraction which forms the hygro bonding may be used.

$$
\varepsilon_{\text {swelling }}=\beta C^{\text {bound water }}
$$

where $\mathrm{C}^{\text {bound water }}$ is the moisture mass for the bond formation per unit volume.

There are a number of experimental techniques for the characterization of the hygroscopic swelling of polymers $[10,32,35,36]$. The aging effect on hygroscopic swelling of a FR4/underfill/chip assembly was also studied. Fig.10 plots the experimental results of the hygroscopic swelling at a constant temperature of $85^{\circ} \mathrm{C}$ as function of time under a humidity condition of $85 \% \mathrm{RH}$ [10]. The red line represents the strain during moisture uptake. It actually consists of two parts: swelling-induced strain and thermal-mismatch induced strain. The blue line is the experimental results for the same assembly without moisture absorption at a constant temperature of $85^{\circ} \mathrm{C}$. Therefore, the actual swelling-induced strain should be the black line in Fig. 10, which is obtained by the red line subtracted from the blue line. It implies that due to the thermal stress relaxation, thermal aging effect must be considered to determine the actual swelling effect.

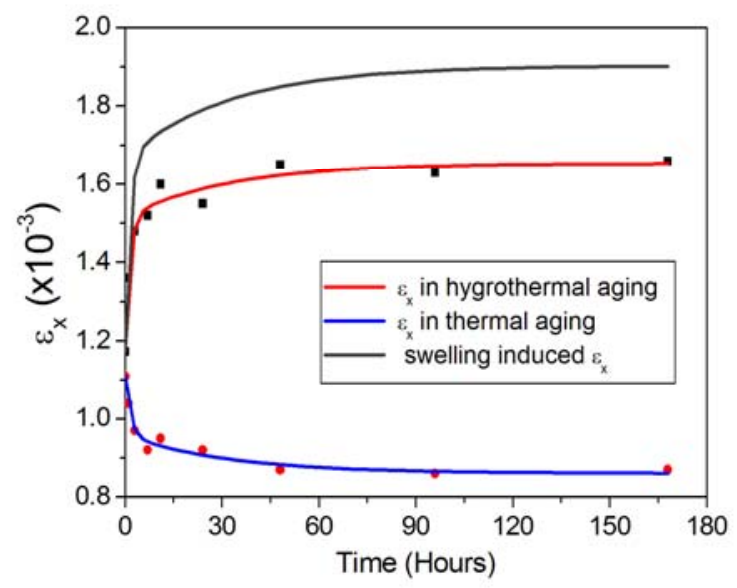

Fig.10. Swelling induced strains from fringe patterns during hygrothermal aging under $85^{\circ} \mathrm{C} / 85 \% \mathrm{RH}$ for a FR4/underfill/chip assembly.

A comprehensive study is developed on the effects of non-uniform moisture distribution and hygroscopic stress in hygroscopic swelling characterization [37,38]. Two different averaged methods are introduced to analyze the experimental data obtained using TMA/TGA techniques with respect to the different reference states chosen, i.e., absolute dry and saturation conditions, respectively. It turns out that these two methods give the upper and lower 
bound estimates of the coefficient of hygroscopic swelling of materials. An integrated finite element analysis methodology, which couples the transient moisture diffusion and the hygroscopic swelling with the temperature change and all nonlinear viscoplastic analysis was reported in ref. [6].

\section{Saturated Moisture Concentration and Solubility}

Saturated moisture concentration $\mathrm{C}_{\mathrm{sat}}$, as defined in the equation (3), is a measure of the moisture absorption capacity under given humidity and temperature conditions. It should be noted that the saturated moisture concentration is a function of both humidity and temperature. According to the definition of the relative humidity

$\mathrm{RH}=\frac{p}{p_{\text {sat }}}=\frac{\text { actural partial water vapor pressure of the air }}{\text { saturated partial water vapor pressure of the air }} \times 100 \%$

The partial water vapor pressure of the air under the given $\mathrm{RH}$ and $\mathrm{T}$ then is

$$
p=R H \times p_{\text {sat }}
$$

where $p_{\text {sat }}$ is the saturated water partial vapor pressure, which can be obtained from steam table. Assume that the Henry's law applies, we have

$$
\begin{gathered}
p=\frac{C_{\mathrm{sat}}}{S} \\
\text { or } S=\frac{C_{\mathrm{sat}}}{p}
\end{gathered}
$$

where $\mathrm{S}$ is the solubility of the material and is a material property. $\mathrm{S}$ is independent of the ambient humidity. From equations (15) and (17), the following relationship can be obtained

$$
C_{\text {sat }}=\left(S \times p_{\text {sat }}\right) R H
$$

Since the solubility and the saturated water vapor pressure is only function of temperature, the saturated moisture concentration is linearly proportional to the relative humidity if the Henry's law is obeyed. Fig. 11 shows the Csat for a BT sample obtained from a RH step scan experiment [5]. It reveals that at a fixed temperature $\left(30^{\circ} \mathrm{C}\right.$ in this case), Csat is linearly proportional to the $\mathrm{RH}$ level. Figure 12 plots the saturated moisture concentration in the BT sample as a function of temperature for two different RH levels. These data were obtained from moisture absorption-desorption experiments. It clearly shows that $C_{\text {sat }}$ in $\mathrm{BT}$ core is essentially temperature independent.

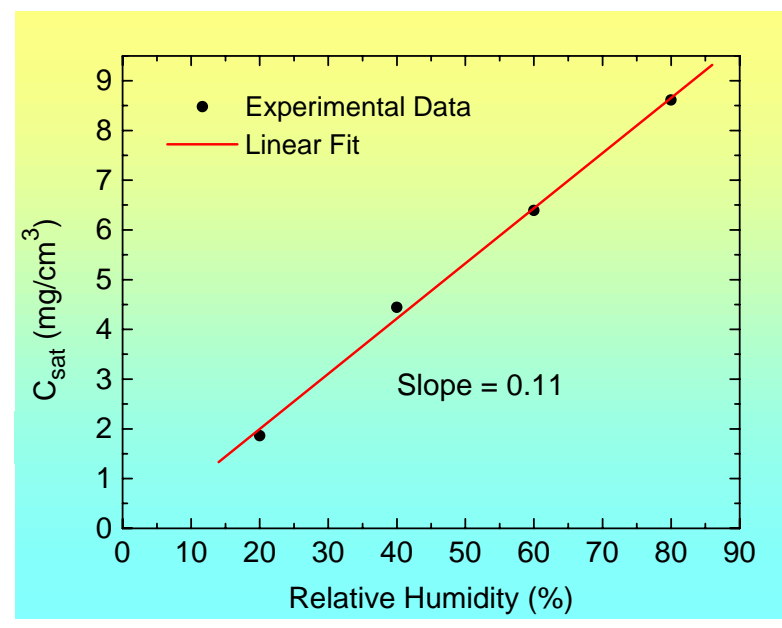

Fig 11. $C_{\text {sat }}$ as a function of relative humidity at $30^{\circ} \mathrm{C}$ for a BT sample [5].

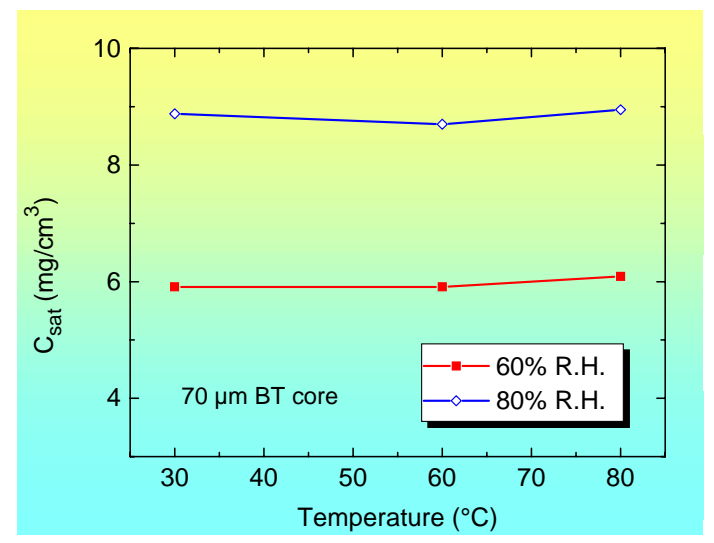

Fig.12. Saturated moisture concentration as a function of temperature for two different RH levels for a BT sample [5].

For many polymer materials applied in microelectronic packaging, Csat is independent of temperature and it depends only on the $\mathrm{RH}$, although exceptions have also been reported. Bao and Yee pointed out that the saturated moisture concentration is related to the heat of moisture absorption [39]:

$C_{\text {sat }}=C_{\text {sat }, r e f} \exp \left[-\frac{\Delta H_{a b s}}{R}\left(\frac{1}{T}-\frac{1}{T_{r e f}}\right)\right]$

where $C_{\text {sat,ref }}$ is the saturated moisture content at a reference temperature $T_{r e f}, \Delta H_{a b s}$ is the heat of moisture absorption, $T$ the absolute temperature and $R$ the universal gas constant. In the absence of chemical reactions between water and the polymer matrix, $\Delta H_{a b s}$ is small. In addition, for practical reasons, the temperature range of most moisture diffusion studies is restricted to between $25^{\circ} \mathrm{C}$ and $90^{\circ} \mathrm{C}$. Therefore, the exponential term in eq. (19) is close to 1 for most polymers that do not react with water, making $C_{\text {sat }}$ nearly temperature independent. 
Fig. 13 plots the saturated moisture concentration for a low-Tg die-attach film as a function of temperature at $60 \%$ RH level. It shows a strong dependency with temperature. This film has a $\mathrm{Tg}$ around $35^{\circ} \mathrm{C}$. It reveals that in the temperature region above the $\mathrm{Tg}$, the saturated moisture concentration depends strongly on the temperature. As a matter of fact, since the saturated moisture concentration has strong dependency on the free volume fraction based on equation (11), it is suggested that the saturated moisture concentration will increase significantly above the glad transition temperature, as illustrated in Fig. 6.

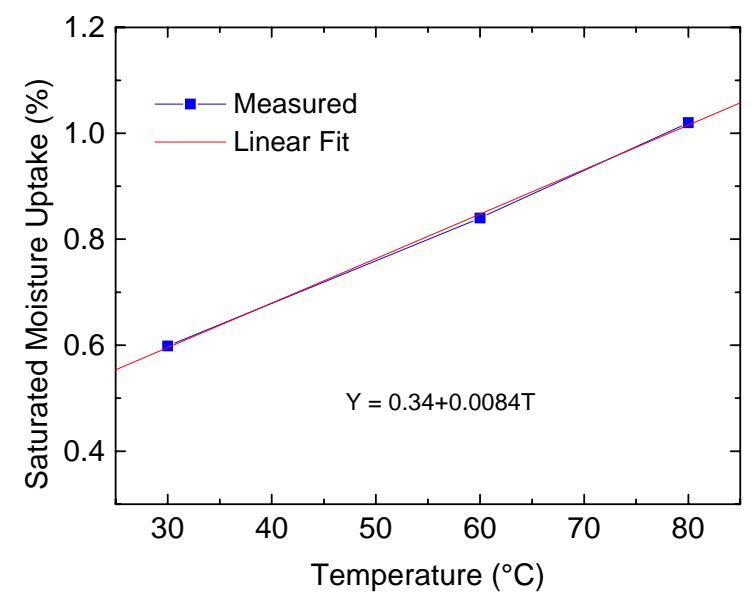

Fig.13. Saturated moisture concentration as function of temperature for a low-Tg film at $60 \% \mathrm{RH}$ level.

The variation of the moisture solubility of polymer material with temperature may be described using Arrhenius equation as following

$$
S=S_{0} \exp \left(\frac{E_{s}}{k T}\right)
$$

where $S_{0}$ is a constant, $E_{s}$ is the activation energy.

Since the saturated vapor pressure increases exponentially with temperature, which may also be described using Arrhenius equation as

$p=p_{0} \exp \left(\frac{E_{p}}{k T}\right)$

From the equations (20) and (21), we have

$$
C_{\text {sat }}=C_{0} \exp \left(\frac{E_{p}+E_{s}}{k T}\right)
$$

It is known that $E_{s}$ is always negative, and $E_{p}$ always positive. Therefore the equation (22) implies that the $\mathrm{C}_{\text {sat }}$ has weak dependency on temperature over a range of temperature below Tg. When the temperature is above Tg, a new set of constants for equation (20) should be used. The weak dependency of the saturated moisture concentration is also consistent with the free-volume theory presented by [21]. It justifies the approximate method to estimate the free volume fraction discussed in the previous section.

\section{Phase Change of Moisture and Vapor Pressure Buildup}

Moisture-induced popcorn failure is a common type of failure that occurs during surface mounting of electronic packages onto printed circuit board. During the reflow soldering process, the entire plastic package is exposed to temperatures as high as $230^{\circ}-260^{\circ} \mathrm{C}$. Under such temperatures, moisture absorbed by package evaporates generating high internal vapor pressures within pores or free volumes.

As we already know, in most of cases, the majority of water molecules neither show significant interaction with other water molecules nor with their environment during preconditioning. Those absorbed water molecules stay in free volume or micro/nano pores and condenses into water liquid form. When the material is heated up during reflow process, the moisture experiences phase transition, which depends on the volume of pores and the temperature. In order to describe the phase change of unbound moisture at elevated temperature, a representative elementary volume (REV) is taken at any considered point in the porous medium domain. The REV is defined in such a way that wherever it is placed within the considered porous medium domain, it always contains both the solid polymer phase and porous/free volume phase. Both the solid phase and porous phase are assumed to be more or less evenly distributed within the REV. Therefore, the free volume fraction $f$ can be introduced, and defined as [20]

$$
f=\frac{V_{f}}{V}
$$

where, $\mathrm{V}_{\mathrm{f}}$ is the current free volume fraction and the $\mathrm{V}$ the total volume of the REV. The instantaneous 'apparent' moisture density $\rho$ in the free volume can then be defined as

$$
\rho=\frac{\Delta M}{V_{f}}=\frac{\Delta M}{V} \frac{V}{V_{f}}=\frac{C}{f}
$$

where $\triangle \mathrm{M}$ is the moisture mass over a REV volume $\mathrm{V}$, and $\mathrm{C}$ is the moisture concentration at a macroscopic level.

When the moisture density $\rho$ in pores is less than the saturated water vapor density $\rho_{\mathrm{g}}$, the moisture is in the single vapor phase, and the vapor pressure can be calculated with the ideal gas law as follows [20, 30, 40,41]

$$
p(T)=\frac{R T}{M M_{H_{2} O} f} \cdot C, \text { when } C(T) / f<\rho_{\mathrm{g}}(T)
$$

where $R$ is the universal gas constant $(=8.314 \mathrm{~J} /(\mathrm{mol} \cdot \mathrm{K}))$, $M M_{H 2 O}$ is the molecular mass of water $(=18 \mathrm{~g} / \mathrm{mol})$. When the moisture density $\rho$ is equal or greater than the saturated water vapor density $\rho_{\mathrm{g}}$ at given temperature, the moisture in the pores is in the mixed liquid-vapor phase. 
Therefore the vapor pressure remains as the saturated vapor pressure as follows

$p(T)=p_{\mathrm{g}}(T)$, when $C(T) / f \geq \rho_{\mathrm{g}}(T)$

where $p_{g}$ is the saturated water vapor pressure. Figure 13 illustrates the two distinct moisture states in free volumes. As shown in Fig 14, only two distinct cases will fully describe the instantaneous moisture states and the phase transition.

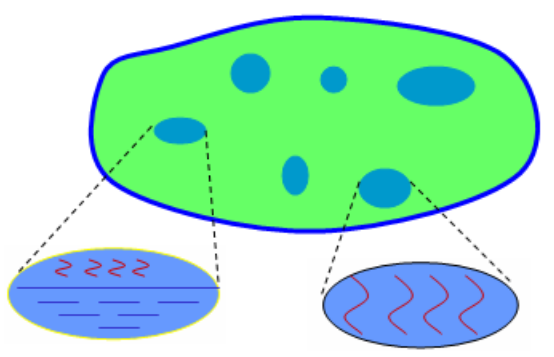

Fig.14. Two distinct instantaneous states of moisture in porous material.

The above analysis has greatly simplified the original vapor pressure model $[20,40]$, in which three distinct cases are identified to describe the moisture states in pores. This modified model $[41,42]$ does not need to relate the current moisture state to a reference moisture state, but yields exact same results with the original model. A simple transformation from the local moisture concentration to vapor pressure can be done through the above vapor pressure model as long as the free volume fraction $f$ and the local moisture concentration are known [43]. However, the free volume fraction changes with the stress state exerted on the material and this will be discussed later.

\section{Interface Discontinuity and Continuity for Moisture Diffusion}

Moisture concentration is discontinuous across the material interface when two materials, which have different saturated moisture concentration $\left(C_{\mathrm{sat}}\right)$, are joined [31]. As shown in Figure 15, for the unsaturated and saturated situations, respectively, the moisture concentration at the interface is given by

$C^{(1)} \neq C^{(2)}$

where $C^{(1)}$ is the moisture concentration at the interface on material 1 (Mat1) side, $C^{(2)}$ is the moisture concentration at the interface on material 2 (Mat2) side.
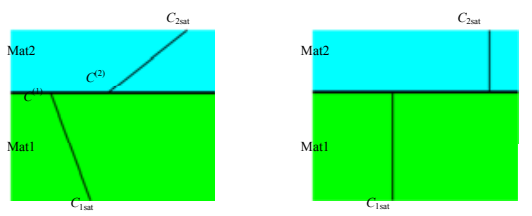

Fig.15. Schematic of moisture distribution in bulk and at interface: (a) unsaturated case, (b) saturated case
However, the interfacial continuity can be expressed by

$C^{(1)} / S_{1}=C^{(2)} / S_{2}$

where $S_{1}$ and $S_{2}$ are the solubility of the Mat1 and Mat2, respectively. From Equations (27) and (28), the interfacial discontinuity can be removed by normalizing the moisture concentration $C$ in the following

$\varphi=C / S$

Galloway et al. [31] demonstrated that in the case of Fickian diffusion, the available commercial finite element software for the transient heat transfer analysis can be used to develop the transient moisture diffusion modeling without any special treatment, as long as the field variable of temperature $T$ in the transient thermal analysis is replaced by the field variable of partial pressure $\varphi$. Similarly, Wong et al. [44] introduced an alternative variable, so-called wetness $w$, which is defined as

$w=C / C_{\mathrm{sat}}$

The wetness $w$ is also continuous at the interface because the saturated moisture concentration $C_{\text {sat }}$ is related to the solubility $S$ by equation (17). When Equation (29) is substituted into the equation (1), and only when the solubility $S$ remains constant for each material during the entire period of time, Equation (1) can be rewritten as

$\nabla^{2} \varphi=\frac{1}{D} \frac{\partial \varphi}{\partial t}$

Similarly,

$\nabla^{2} w=\frac{1}{D} \frac{\partial w}{\partial t}$

However, when the temperature $T$ varies with the time $t$, the solubility $S$ varies with the time $t$. The simple transformation from Equation (1) to Equation (32) does not exist, but is given by

$\nabla^{2} \varphi=\frac{1}{D} \frac{\partial \varphi}{\partial t}+\frac{\varphi}{D \cdot S} \frac{\partial S}{\partial t}$

in which

$\frac{\varphi}{D \cdot S} \frac{\partial S}{\partial t} \neq 0$

when the solubility $S$ is not constant under the varying ambient temperature and humidity conditions. For the Wong's normalization approach, the governing differential equation of the moisture diffusion is changed to

$\nabla^{2} w=\frac{1}{D} \frac{\partial w}{\partial t}+\frac{w}{D \cdot C_{s a t}} \frac{\partial C_{s a t}}{\partial t}$

in which 


$$
\frac{w}{D \cdot C_{s a t}} \frac{\partial C_{s a t}}{\partial t} \neq 0
$$

when the saturated moisture concentration $C_{\text {sat }}$ becomes dependent on the time $t$. Equations (33) and (35) indicate the simple thermal-moisture analogy does not apply when ambient temperature or humidity conditions are dependent on time (or temperature). Equation (35) can be reduced to the Equation (1) only when the saturated moisture concentration is independent on temperature (thus time $t$ ).

In order to correctly perform the moisture diffusion modeling under the varying temperature and humidity conditions, a direct concentration approach (so-called DCA) is introduced [41,42]. Different from the normalization methods, the moisture concentration $C$ is directly used as the basic field variable. Additional treatment is necessary to ensure the following continuous conditions at interfaces

$$
\begin{aligned}
& C^{(1)} / S_{1}(t)=C^{(2)} / S_{2}(t) \\
& D_{1}(t) \frac{\partial C^{(1)}}{\partial n}=D_{2}(t) \frac{\partial C^{(2)}}{\partial n}
\end{aligned}
$$

where $D_{1}(t)$ and $D_{2}(t)$ are the moisture diffusivity of the Mat1 and Mat2 respectively, and $n$ is the normal direction of the location at the interface. In order to satisfy the interfacial continuity conditions, two separate sets of nodes can be applied at a bi-material interface to represent the discontinuity of the moisture concentration, as shown in Figure 16. According to the variational principle, Equation (38) will be automatically satisfied through the finite element formulation as long as the Equation (37) is guaranteed.

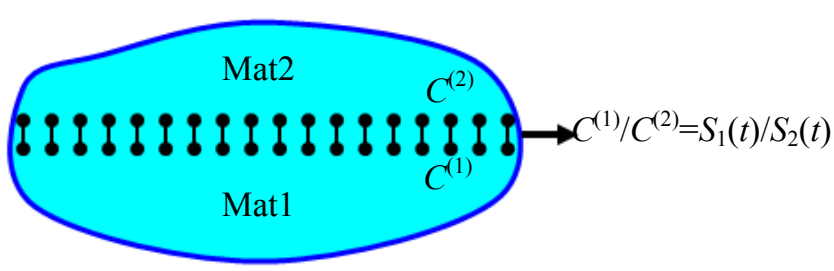

Fig.16. Special treatment at a bi-material interface in the direct concentration approach.

In general moisture diffusion is a 'slow' process compared to the heat conduction process for the same material. For example, for a polymer film with $2 \mathrm{~mm}$ thickness, the thermal and moisture diffusivities are $4.22 \times 10^{-3} \mathrm{~cm}^{2} / \mathrm{s}$ and $2.85 \times 10^{-8} \mathrm{~cm}^{2} / \mathrm{s}$, respectively. Fig. 17 plotted the results for the local moisture concentration and temperature at $x=h$ as the function of time [30]. For level $3\left(30^{\circ} \mathrm{C} / 60 \% \mathrm{RH}\right)$, the slab is far from the saturated after 500 hours. The nearly saturated state is reached for level $2\left(85^{\circ} \mathrm{C} / 60 \% \mathrm{RH}\right)$ and level 1 $\left(85^{\circ} \mathrm{C} / 85 \% \mathrm{RH}\right)$, respectively. However, the heat transfer reaches the equilibrium much faster than moisture diffusion. This implies that the moisture diffusion can be decoupled from the heat transfer due to the difference in orders in reaching equilibrium. However it has been found that for the ultra thin packages, the diffusion of moisture during reflow process is as fast as heat transfer therefore, the coupled analysis is necessary [41].
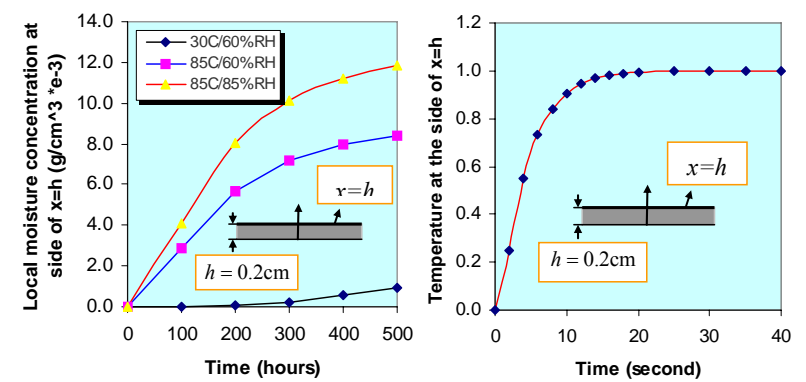

Fig.17. comparison of heat transfer and moisture diffusion for a polymer material of $2 \mathrm{~mm}$ thickness.

\section{Governing Equations for a Deforming Polymer with Moisture Considering Phase Transition}

In this section the governing equations for a deforming polymer with moisture are developed. The general case of the moisture phase transition, which is described in previous section, is considered. The governing equations are developed on the lines of soil or rock mechanics with multiphase flow. The solid phase is assumed to comprise a porous skeleton of polymers surrounded by moisture. The moisture flow is neglected here, whilst an all-round vapor pressure is exerted on the solid phase. The small strain theory is considered here for clarity, but the theory can be extended to largedeformation case. It is assumed that a vapor pressure by moisture, i.e. $p$, causes only a uniform, volumetric strain by compressing the solids and that the major deformation of the porous skeleton is governed by the effective stress $\sigma$. This is defined as follows, with the sign convention that tension is positive,

$\boldsymbol{\sigma}=\boldsymbol{\sigma}^{\prime}-\mathbf{m} p$

where $\sigma$ is the total stress and $\mathbf{m}$ is equal to unity for the normal stress components and zero for the shear stress components. Equation (39) can be obtained by micromechanics approach. At each point in the domain, the variables are in fact average values over a representative elementary volume (REV) around any considered point in the porous medium domain. As long as the REV is independent of time and of location in the porous medium, the averaged equations obtained are independent of the geometry of the REV. The volume V of a REV is composed by the sum of the volume of solid phase and free volume

$V=V_{s}+V_{f}$

where $V_{s}$ is solid phase volume and $V_{f}$ is the free volume. The (averaged) total stress vector at macroscopic level may be expressed in terms of intrinsic phase averages 


$$
\begin{aligned}
\langle\boldsymbol{\sigma}\rangle & =\frac{1}{V} \int_{V} \sigma d V=\frac{1}{V}\left[\int_{V_{s}} \sigma d V+\int_{V_{f}} \sigma d V\right] \\
& =\frac{V_{s}}{V}\left\langle\sigma_{s}\right\rangle^{s}+\frac{V_{f}}{V}\left[\frac{1}{V_{f}}\left\langle\sigma_{f}\right\rangle^{f}\right]
\end{aligned}
$$

where

$$
\left\langle\sigma_{\pi}\right\rangle^{\pi}=\frac{1}{V_{\pi}} \int_{V} \sigma_{\pi} d V
$$

which, presents the average over one-phase. Then we have

$$
\langle\boldsymbol{\sigma}\rangle=(1-f)\langle\boldsymbol{\sigma}\rangle^{s}-f \mathbf{m}\langle p\rangle=\boldsymbol{\sigma}^{\prime}-\mathbf{m}\langle p\rangle
$$

This is how we obtained equation (39). For clarity, we do not use the symbol $<>$, but one should always keep in mind that the quantities are in average at macroscopic level. In equation (43),

$$
\boldsymbol{\sigma}^{\prime}=(1-f)\left(\langle\boldsymbol{\sigma}\rangle^{s}+\mathbf{m}\langle p\rangle\right)
$$

is the strain-producing stress in the solid skeleton, the effective stress. According to Terzaghi's definition it the sum of the pressure and average stress in the solid phase.

The constitutive equation relating this effective stress to the strains of the skeleton is now independent of the vapor pressure $\mathrm{p}$, and for a general nonlinear material can be written in a tangential from, thus allowing plasticity to be incorporated. The expression is written in general from as

$$
d \boldsymbol{\sigma}^{\prime}=\mathbf{D}_{\mathrm{T}}\left(d \boldsymbol{\varepsilon}-d \boldsymbol{\varepsilon}_{\mathrm{c}}-d \boldsymbol{\varepsilon}_{\mathrm{p}}-d \boldsymbol{\varepsilon}_{\mathbf{0}}\right)
$$

where $\mathrm{d} \varepsilon$ represents the total strain of the skeleton, and represents the overall volumetric strains caused by uniform compression of the particles (as opposed to skeleton) by the pressure of the moisture, with $\mathrm{K}_{\mathrm{s}}$ being the bulk modulus of the solid phase. The volumetric strain is relatively insignificant when polymer behaves as rubber where the material is nearly incompressible.

The matrix $\mathbf{D}_{\mathrm{T}}$ are dependent on the level of effective stresses and also, if strain effects are considered, on the total strain of the skeleton $\varepsilon$. The equilibrium equation relating the total stress to the body forces and the boundary traction specified at the boundary of the domain is formulated in terms of the unknown displacement vector $u$. When the effective stress relationship is incorporated, the following equation is obtained

$$
\begin{aligned}
& \int_{\Omega} \delta \boldsymbol{\varepsilon}^{\mathbf{T}} \mathbf{D}_{\mathbf{T}} \frac{\partial \boldsymbol{\varepsilon}}{\partial t} d \Omega-\int_{\Omega} \delta \boldsymbol{\varepsilon}^{\mathbf{T}} \mathbf{m} \frac{\partial p}{\partial t} d \Omega+\int_{\Omega} \delta \boldsymbol{\varepsilon}^{\mathrm{T}} \mathbf{D}_{\mathbf{T}} \mathbf{m} \frac{\partial p}{\partial t} \frac{1}{3 K_{s}} d \Omega \\
& -\int_{\Omega} \delta \boldsymbol{\varepsilon}^{\mathrm{T}} \mathbf{D}_{\mathbf{T}} \frac{\partial \boldsymbol{\varepsilon}_{0}}{\partial t} d \Omega-\int_{\Omega} \delta \boldsymbol{\varepsilon}^{\mathbf{T}} \mathbf{D}_{\mathbf{T}} \frac{\partial \boldsymbol{\varepsilon}_{c}}{\partial t} d \Omega-\frac{\partial \hat{\mathbf{f}}}{\partial t}=0
\end{aligned}
$$

$$
d \widehat{\mathbf{f}}=\int_{\Omega} \delta \mathbf{u}^{\mathrm{T}} d \mathbf{b} d \Omega+\int_{\Omega} \delta \mathbf{u}^{\mathrm{T}} d \mathbf{t} d \Gamma
$$

If the pressure is known, then the displacements and stresses within the system can be obtained from this equation. However, in general the pressure field is coupled with the strain changes, which is described previously. The above equation must be solved together with equations (25) and (26).

In equation (46) there are two terms related to the vapor pressure. Previously, the concept of vapor pressure induced expansion was introduced [20]. An integrated vapor pressure, hygroswelling and thermo-mechanical stress modeling methodology during reflow with interfacial fracture mechanics analysis modeling was developed to study the vapor pressure effect [45]. It can be seen that such an effect corresponds to the third term on the left side of equation (46). When the material is incompressible, this term vanishes. However, the vapor pressure affects the polymer matrix deformation through the second term, which was not considered in the previous approach.

\section{Discussions and Conclusions}

This paper addressed some fundamental concepts and understandings on the effect of moisture for polymer mechanical behaviors. 'Two-stage' sorption theory is introduced to describe the non-Fickian diffusion. The two-stage sorption is characterized by a rapid initial uptake to a quansi-equilibrium, followed by a slower approach to a final, true equilibrium. The second stage involves a further increase in surface concentration due to relaxation of the polymer. For practical reasons, if the attention is focused on the moisture diffusion process when the second stage has not taken into effect, the Fickan behavior can be applied.

There are two different diffusion mechanisms involved in the transport of moisture: the transfer across the surface and the transfer through bulk. Water sorption appears to be different from the moisture sorption. For some water-proof coating film, the water liquid molecules can be prevented from penetrating through the surface. However, this has never been effective for the water vapor molecules into the material.

Moisture is absorbed into polymeric materials in two ways. The first is as free or 'unbound' water liquid or vapor, which collects at micro- or nano- pores, free volumes, interfaces, and micro- or macro voids. More than $90 \%$ of the absorbed moisture stays in bound water state. Moisture can also be absorbed by water-polymer affinity for each other due to the availability of hydrogen bonding sites along the polymer chains and interfaces, which are known as 'bound water'. The formation of hydorgen bond with polymer materials casues the hygroscopic swelling of material, while the unbound water liquid/vapor fills in free volumes, which does not cause swelling if the vapor pressure is low at lower temperatures. The hygroscopic swelling is usually much 
smaller than the volume occupied by the absorbed moisture, which indicates that the moisture in free volumes does not cause the material's swelling.

The saturated moisture concentration is strongly related to the free volume fraction and it has been found that the saturated moisture concentration has weak temperature dependancy over a temprature range below the glass transition temperature. However, the saturated moisture content may increases significantly with temperature when the temperature is above $\mathrm{Tg}$.

A vapor pressure model, which allows the whole-field vapor pressure calculations, is presented, and the governing equations for a deforming polymer with the consideration of moisture are developed.

\section{References}

1. X.J. Fan, "Moisture related reliability in electronic packging", 2005/2006/2007 ECTC Professional Development Course Note, 2005/2006/2007

2. G.Q. Zhang, W.D. van Driel, and X.J. Fan, "Mechanics of Microelectronics", Springer, 2006

3. A.A.O. Tay, and T.Y. Lin, "Influence of Temperature, Humidity and Defect Location on Delamination in Plastics Packages", IEEE Transactions on Components, Packaging and Manufacturing Technologies, Part A, 22, No. 4, pp. 512-518, 1999

4. S. Liu and Y. H. Mei, "Behavior of Delaminated Plastic IC Packages Subjected to Encapsulation Cooling, Moisture Absorption, and Wave Soldering", IEEE Transactions on Components, Packaging, and Manufacturing Technologies, Part A, Vol. 18, No. 3, 1995

5. Y. He, and X.J. Fan, "In-situ characterization of moisture absorption and desorption in a thin BT core substrate", Electronic Components and Technology Conference, pp. 1375-1383, 2007

6. X.J. Fan, J. Zhou, and A. Chandra "Package integrity analysis with the consideration of moisture effects", Electronic Components and Technology Conference, 2008

7. X.Q. Shi, Y.L. Zhang, W. Zhou, and X.J. Fan, "Effect of hygrothermal aging on interfacial reliability of silicon/underfill/FR-4 assembly", IEEE Transactions of Components and Packaging Technologies, 2008 (in press)

8. E. Prack and X.J. Fan, "Root cause mechanisms for delamination/cracking in stack-die chip scale packages", International Symposium on Semiconductor Manufacturing (ISSM), 2006, September 25 - 27, Tokyo, Japan.

9. X.J. Fan, G.Q. Zhang, and L.J. Ernst, "Interfacial delamination mechanisms during reflow with moisture preconditioning", IEEE Transactions of Components and Packaging Technologies, 2008 (in press).
10. X.Q. Shi and X.J. Fan, "Wafer-level film selection for stacked-die chip scale packages", Electronic Components and Technology Conference, pp. 1731-1736, 2007.

11. T. Ferguson and J. Qu, "Moisture absorption analysis of interfacial fracture test specimens composed of no-flow underfill materials", Journal of Electronic Packaging, Vol. 125, pp 24-30, 2003.

12. S. Luo and C.P. Wong, "Moisture absorption in uncured underfill materials", IEEE Transactions of Components and Packaging Technologies, $\mathrm{Vol}$ 27, No.2, 345-351, 2004

13. S. Luo and C.P. Wong, "Influence of temperature and humidity on adhesion of underfills for flip chip packaging", IEEE Transactions of Components and Packaging Technologies ,Vol. 28, No.1, 88-94, 2005

14. G.K. van der Wel and O.C.G. Adan, "Moisture in organic coatings - a review", Progress in Organic Coatings, 37, 1-14, 1999

15. L. Pel, H. Brocken, K. Kopinga, Int. J. Heat Mass Transfer 39 (1996), 127

16. J.S. Vrentas, C.M. Vrentas, W.J. Huang, J. Appl. Polym. Sci. 64, p. 2007, 1997

17. A.R. Berens, H.B. Hopfenberg, Polymer 19 (1978) 489

18. Y.-M. Sun, H.-L. Lee, Polymer 37 (1996) 3921

19. C.Q. Cui, X.J. Fan, Unpublished report on the study of water-proof coating film for preventing moisture-induced failures for QFN packages, 1999

20. X.J. Fan, J. Zhang, G.Q. Zhang and L.J. Ernst, "A micromechanics based vapor pressure model in electronic packages", ASME Journal of Electronic Packaging, 127 (3), pp. 262-267, 2005.

21. J.L. Duda, J.M. Zielinski, in: P. Neogi (Ed.), "Diffusion in Polymers", Ch. 3, Marcel Dekker, New York, 1996.

22. H. Ardebili, E.H. Wong, and M. Pecht, "Hygroscopic swelling and sorption characteristics of epoxy molding compounds used in electronic packaging", IEEE Trans. Comp. Packag. Technol., Vol. 26, No. 1 (2003) pp. 206-214.

23. M. G. McMaster and D. S. Soane, "Water sorption in epoxy thin films", IEEE Transactions on Components, Hybrid, and Manufacturing Technology, 12(3), 373-386, 1989

24. R. Delasi and J.B. Whiteside, in Advances Composite Materials - Environmental Effects, J.R. Vinson Ed., ASTM STP-658, 1978

25. E.L. Mckague, J.D. Reynolds, and J.E. Halkias, Trans Amer Soc Mech Eng, 98, p.92, 1976 
26. Paul A. Webb, "An introduction to the physical characterization of materials by mercury intrusion porosimetry with emphasis on reduction and presentation of experimental data", Micromeritics Instrument Corp, 2001

27. P. Koning, X. J. Fan, unpublished report on mercury intrusion porosimetry measurement for several kinds of polymer materials, 2006

28. X.J. Fan, Delamination/cracking mechanism study for ultra-thin stacked-die chip scale packages, Intel Conference on Manufacturing Excellence (IMEC), 2006, San Diego, CA

29. JEDEC Moisture sensitivity test standard

30. X.J. Fan and T. B. Lim, "Mechanism analysis for moisture-induced failures in IC packages", ASME 1999 International Mechanical Engineering Congress, IMECE/EPE-14, 1999.

31. J.E. Galloway and B.M. Miles, "Moisture absorption and desorption predictions for plastic ball grid array packages", IEEE Transactions on Components, Packaging and Manufacturing Technology, Part A, 20(3), pp. 274-279, 1997.

32. Eric Stellrecht, Bongtae Han, and Michael G. Pecht, "Characterization of Hygroscopic Swelling Behavior of Mold Compounds and Plastic Packages", IEEE Transactions on Components and Packaging Technologies, 27(3), 499-505, 2004

33. D. L. Vanderhart, M. A. Schen, and G. T. Davis, "Partitioning of water between voids and the polymer matrix in a molymer compound by proton NMR: The role of larger voids in the phenomena of popcorning and delamination," Int. J. Microcircuits Electron. Packag., vol. 22, no.4, 1999.

34. C. Toprak, J.N. Agar, and M. Falk, "State of water in cellulose acetate membranes," J. Chem. Soc., Faraday Trans. I, vol. 75, p. 803, 1979

35. Wong, E.H., Chan, K.C., Rajoo, R., Lim, T.B., "The mechanics and impact of hygroscopic swelling of polymeric materials in electronic packaging," Proc. 50 $0^{\text {th }}$ Electron. Comp. Technol. Conf., Las Vegas, NV, 2000, pp. 576-580, 2000

36. Tee, T. Y., Kho, C. 1., Yap, D., Toh, C., Baraton, X., Zhong, Z., "Reliability assessment and hygroswelling modeling of FCBGA with noflow underfill", Microelectronics Reliablity, pp. 741-749, 2003

37. J. Zhou, "Investigation of non-uniform moisture distribution on determination of hygroscopic swelling coefficient and finite element modeling for a flip chip package, IEEE Transactions of Components and Packaging Technologies, 2008 (in press).
38. J. Zhou, "Analytical and numerical bound analysis of hygroscopic swelling characterization", ECTC 2006

39. Li-Rong Bao, A. F. Yee, Effect of Temperature on Moisture Absorption in a Bismaleimide Resin and Its Carbon Fiber Composites, Polymer, Vol. 43, No. 14 (2002), pp. 3987-3997

40. Xuejun Fan; G. Q. Zhang; W. D. van Driel; L. J. Ernst, Analytical solution for moisture-induced interface delamination in electronic packaging, Electronic Components and Technology Conference, 2003, May 27-30, 2003 Page(s):733-738

41. B. Xie, X.Q. Shi, X.J. Fan, and H. Ding, "Direct concentration approach of moisture diffusion and whole field vapor pressure modeling for reflow process", submitted, 2008

42. B. Xie, X. Q. Shi, and X.J. Fan, Sensitivity investigation of substrate thickness and reflow profile on wafer level film failures in 3D chip scale packages by finite element modeling, 57th Electronic Components and Technology Conference 2007, ECTC '07, 2007, p 242-248

43. T.Y. Tee, X.J. Fan and T. B. Lim, "Modeling of whole field vapor pressure during reflow for flip chip and wire-bond PGBA Packages", $1^{\text {st }}$ International Workshop on Electronic Materials \& Packaging, 1999

44. E. H. Wong, Y. C. Teo, and T. B. Lim, "Moisture diffusion and vapor pressure modeling of IC packaging", $48^{\text {th }}$ Electronic Components and Technology Conference, pp.1372-1378, 1998.

45. T. Y. Tee and Z. W. Zhong, "Integrated vapor pressure, hygroswelling and thermo-mechanical stress modeling of QFN package during reflow with interfacial fracture mechanics analysis", Microelectronics Reliability, Vol. 44(1), pp. 105114, 2004. 